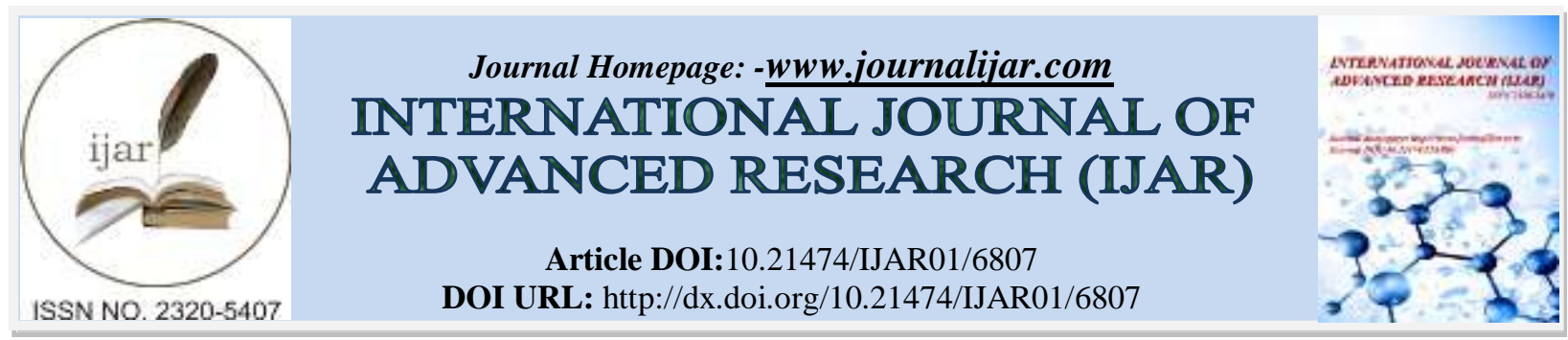

RESEARCH ARTICLE

\title{
PULSATING ABDOMEN: DIAGNOSTIC DILEMMA- A CASE REPORT.
}

\section{Gargi $\mathbf{G}^{1}$, Kumar $\mathrm{M}^{2}$, Singh $\mathrm{G}^{3}$, Saini $\mathrm{A}^{4}$ and Goyal $\mathrm{SK}^{5}$.}

1. Senior Resident, Department of Medicine, Dr. Rajendra Prasad Govt Medical College (RPGMC), Kangra at Tanda, Himachal Pradesh, India.

2. Assistant Professor, Department of Cardiology, Dr. Rajendra Prasad Govt Medical College (RPGMC), Kangra at Tanda, Himachal Pradesh, India.

3. Junior Resident, Department of Medicine, Dr. Rajendra Prasad Govt Medical College (RPGMC), Kangra at Tanda, Himachal Pradesh, India.

4. Senior Resident, Department of ENT, Dr Rajendra Prasad Govt Medical College, Kangra at Tanda (RPGMC), Himachal Pradesh, India.

5. Independent Researcher, Tanda, Kangra, Himachal Pradesh, India.

\section{Manuscript Info}

Manuscript History

Received: 21 January 2018

Final Accepted: 23 February 2018

Published: March 2018

Keywords:-

Twiddler's syndrome.

\begin{abstract}
It is rare to find a patient with pulsating abdomen in the emergency room. We report a patient with pulsating abdomen having a rare diagnosis Twiddler's syndrome. Twiddler's syndrome is a permanent malfunction of the cardiac pacemaker due to the patient's manipulation of the pulse generator. Due to this, the leads are dislodgedand can stimulate ipsilateral phrenic nerve leading to rhythmic diaphragmatic pacing and sensation of pulsating abdomen. This is a rare case report of an elderly male with Twiddler's syndrome resulting in pacemaker malfunction secondary to lead retraction. He subsequently underwent lead repositioning and appropriate counseling of not to manipulate the pacemaker pulse generator within its skin pocket.
\end{abstract}

Copy Right, IJAR, 2018,. All rights reserved.

\section{Introduction:-}

Very rarely a physician in the emergency room encounters a patient with a pulsating abdomen. A common differential diagnosis which strikes your mind is some vascular mass like an aortic aneurysm. We report here a case with pulsating abdomen with a diagnostic dilemma finally having a cardiac cause: Twiddler's syndrome. This syndrome was first described in 1968 by Byliss and refers to permanent malfunction of a pacemaker due to the patient's manipulation of the pulse generator [1]. The process usually starts with deliberate or subconscious manipulation of the pacemaker's pulse generator in a capacious pocket. Due to this, the leads are dislodged and the ventricular pacing stops. These dislodged leads can stimulate ipsilateral phrenic nerve leading to rhythmic diaphragmatic pacing and sensation of pulsating abdomen.

\section{Case Report:-}

A 70-year-old male patient presented to the emergency department with a history of syncope and continuous uncomfortable pulsations in his abdomen for several hours (video 1). On arrival, he was found to have rhythmic twitching of the right arm. He was admitted one month back with a similar history of syncope and was diagnosed as symptomatic complete heart block. Permanent transvenous RV endocardial pacing through left subclavian was done 
(VVIR mode and rate 60/min). After discharge from the hospital, he was admitted to repeatedly manipulating the pacemaker in his left pectoral region. This time, physical examination revealed heart rate of 32 beats/minutes, blood pressure of $92 / 70 \mathrm{mmHg}$. He had no cognitive deficit. ECG showed complete heart block with pacemaker spikes and failure to capture (fig 1). Chest X ray showed coiled and retracted right ventricular lead and rotated pulse generator (fig 2). The patient underwent successful lead repositioning with strong counseling to avoid further manipulation of the device.

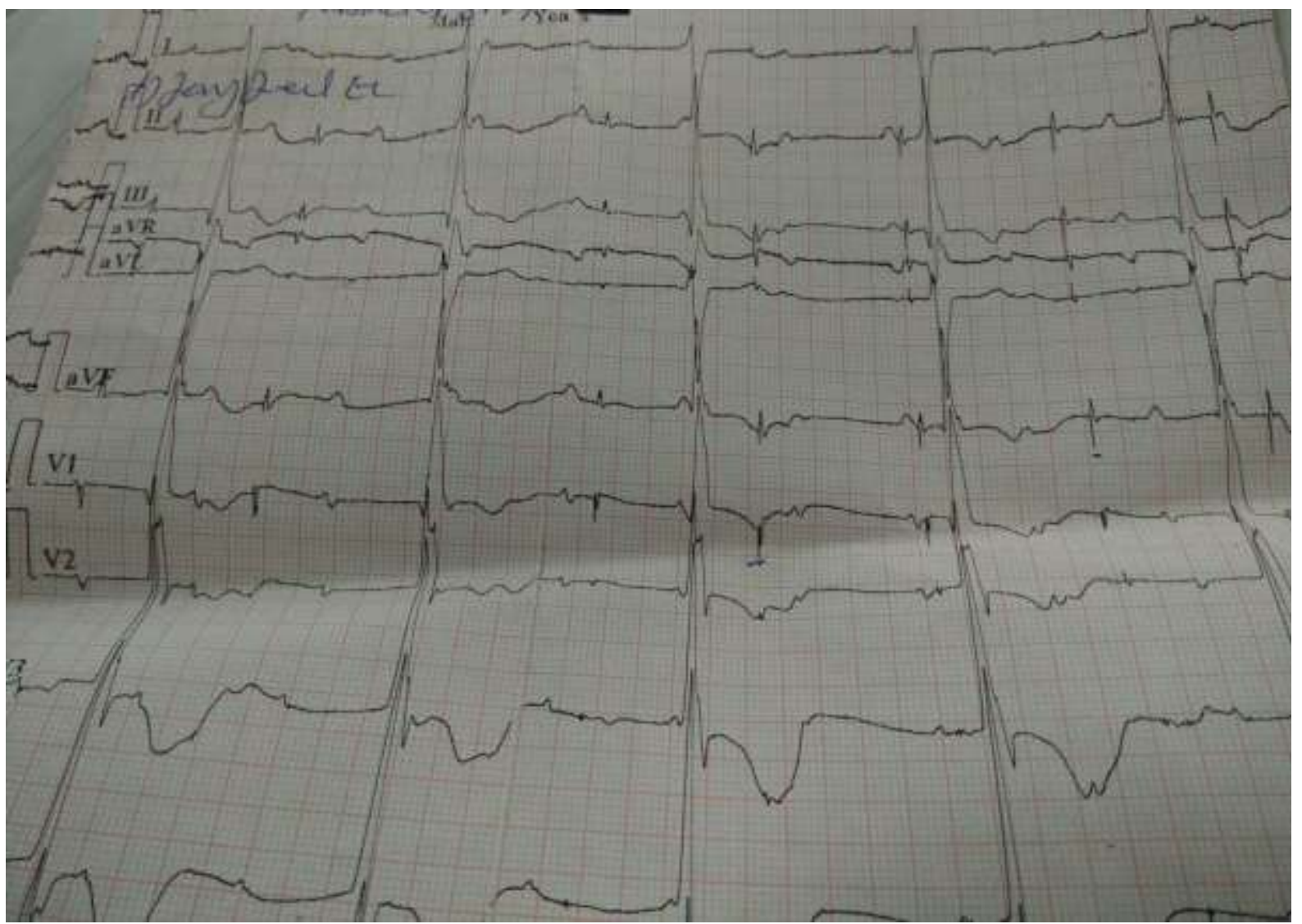

Fig 1:-ECG 


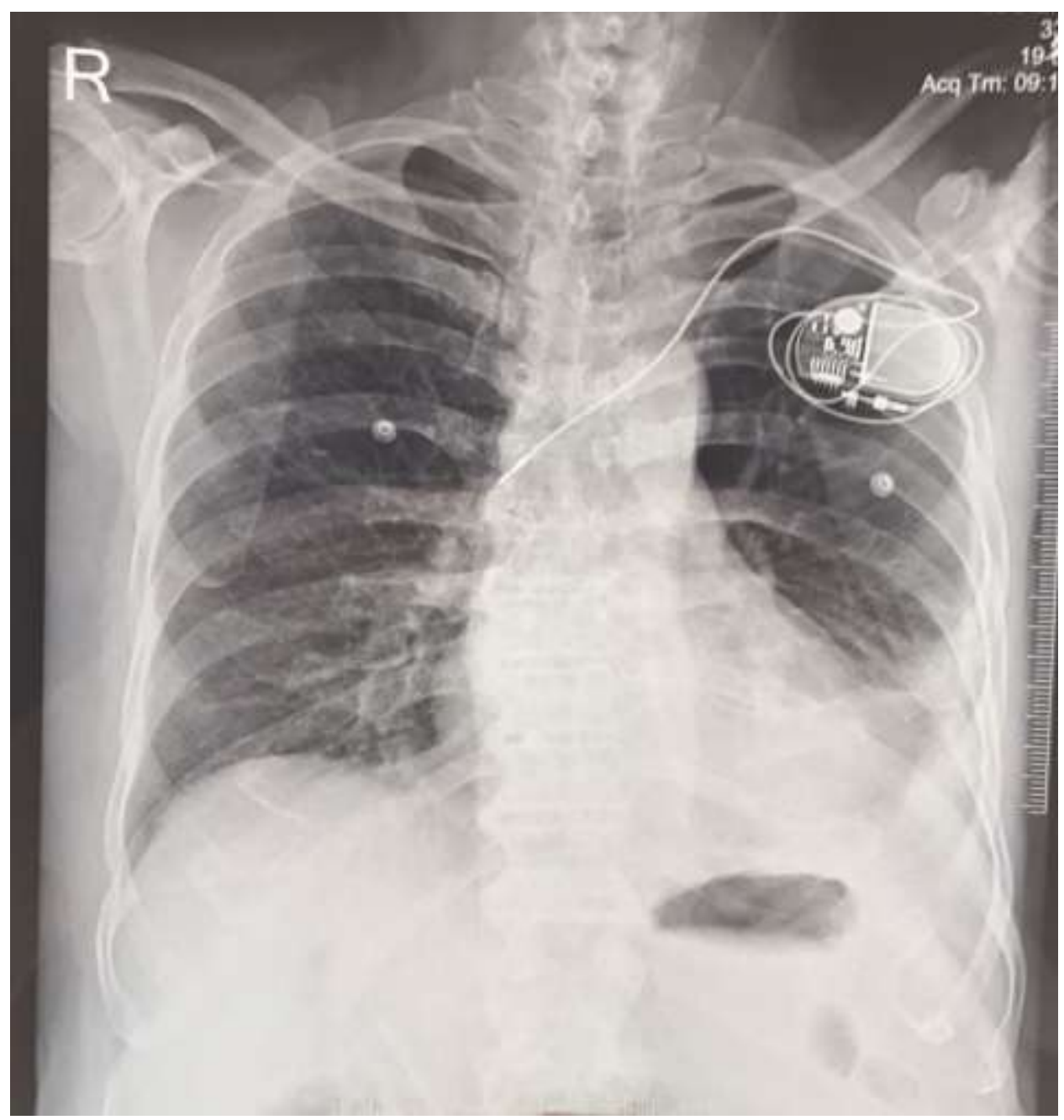

Fig 2:- Chest X-ray

\section{Discussion:-}

Twiddler's syndrome is a rare but potentially lethal complication of cardiac pacemaker treatment. The pacemaker Twiddler's syndrome has an estimated frequency of around $0.007-7 \%$ [2,3].The majority of cases are diagnosed with in the first year of implant although it can occur any time after device implantation[4,5].Our patient presented after four weeks of permanent pacemaker implantation. The earliest reported case of this syndrome is at about 17 hours [2]. Late pacemaker Twiddler's syndrome has also been reported by some authors [6].

The risk factors for the condition include female gender, obesity, elderly age group, impaired cognition and a smaller sized implanted device relative to its pocket $[2,7,8]$. An associated increased laxity of the subcutaneous tissues particularly in elderly patients facilitates further dislodgement of the device [4,5].

The majority of patients deny manipulating the device [9]. Our patient; however, admitted manipulating the device which could have been avoided by better patient education. This manipulation of the device by the patient may have dislodged the leads which may have stimulated the ipsilateral phrenic nerve causing diaphragmatic contractions, occasional spasms of involuntary respiration or hiccups and a sense of pulsating abdomen. Further coiling and withdrawal of the lead can cause stimulation of brachial plexus, resulting in rhythmic arm twitching $[1,5,9]$.

This syndrome can be easily diagnosed on plain $\mathrm{X}$ - ray chest which gives a clear image of lead coiling and device rotation [5]. Some people often ignore this simple, rapid and cheap investigation and proceed to Holter monitoring. Minimizing the pocket size and suturing the device generally prevent the development of this syndrome. Some authors have advocated the use of a Dacron patch, which stabilizes the pulse generator by promoting tissue in growth, in all cases of device implantation [10]. Active fixation of leads is also encouraged. 


\section{Conclusion:-}

Twiddler's syndrome is a rare complication of permanent pacemaker implantation with potential catastrophic consequence generally diagnosed with in the first year of implantation. Treatment involves repositioning of dislodged leads and suture fixation of lead and pulse generator within its pocket.

\section{References:-}

1. Bayliss CE, Beanlands DS, Baird RJ. The pacemaker-twiddler's syndrome: a new complication of implantable transvenous pacemakers. Can Med Assoc 0.0J. 1968;99:371-373.

2. Fahraeus T, Hijer CJ. Early pacemaker Twiddler syndrome. Europace. 2003;5:279-81.

3. Hill PE. Complications of permanent transvenous cardiac pacing: A 14-year review of all transvenous pacemakers inserted at one community hospital. Pacing Clin Electrophysiol. 1987;10:564-70.

4. Sharifi M, Inbar S, Neckels B, Shook H. Twiddling to the extreme: development of twiddler syndrome in an implanted cardioverter-defibrillator. J Invasive Cardiol. 2005;17:195-196

5. DeMarco DC, Xuereb RG. 'Twiddling' of the pacemaker resulting in lead dislodgement. Malta Med J. 2009;21(3):38-41.

6. Dursun I, Yesildag O, Soylu K, Yilmaz O, Yasar E, Meric M. Late pacemaker Twiddler syndrome. Clin Res Cardiol. 2006;95:547-9.

7. Gupta R, Lin E. Twiddler syndrome. J Emerg Med. 2004;26:119-20.

8. Castilo R, Cavusoglu E. Twiddler's syndrome: An interesting cause of pacemaker failure. Cardiology. 2006;105:119-21.

9. Nicholson WJ, Tuohy KA, Tilkemeier P. Twiddler's syndrome. N Engl J Med. 2003;348:1726-7.

10. Furman S. Defibrillator Twiddler's syndrome. Ann Thorac Surg. 1995;59:544-51. 